\title{
Different vectors used to transform and clone of nonstructural NS1 gene of Influenza B in Escherichia coli
}

\author{
A.A. Dawood \\ Department of Anatomy, College of Medicine, University of Mosul, Mosul, Iraq \\ aliadeldawood@gmail.com
}

(Received November 9, 2018; Accepted December 21, 2018)

\begin{abstract}
Flu is a highly contagious and common illness caused by influenza A, B, and C viruses. The aim of the present study was to investigate the transformation and cloning of NS1B gene with pET-32a, pET-32b and pQE-81L in Escherichia coli BL21(DE3) and DH5 $\alpha$. pUC57-NS1B synthetic gene was transform and clone in Escherichia coli BL21(DE3). Isolation, single digestion and ligation with pET-32b using HindIII restriction enzyme. Amplification of recombinant DNA was done with conventional PCR after transformation. Screening with IPTG of colonies. Gel electrophoresis was done for each step of cloning after isolation. Isolation, double digestion and ligation with pET-32a and pQE-81L using SacI, PstI and HindIII respectively. Recombinant DNA was attempted to be transformed into E. coli strains BL21 (DE3) and DH5 $\alpha$. pUC57 plasmid carrying NS1B gene was successful transformed and isolated from E. coli BL21 (DE3). Designed primers used for PCR of NS1B showed successful amplification. First screening of pET-32b-NS1B colonies using white/blue method, cloning NS1B into pET-32b using single restriction digestion with HindIII, pET-32a using double restriction digestion with SacI and HindIII and $\mathrm{pQE}-81 \mathrm{~L}$ using double restriction digestion with PstI and HindIII gave unexpected result. This result may relate to religation of digested vector for single digestion and uncompleted digestion for vectors of double restriction digestion. Current study has suggested that recombinant NS1B gene can be cloned using single digestion with other expression vectors.
\end{abstract}

Keyword: Nonstructural protein, Influenza B, Polymerase chain reaction Available online at http://www.vetmedmosul.com

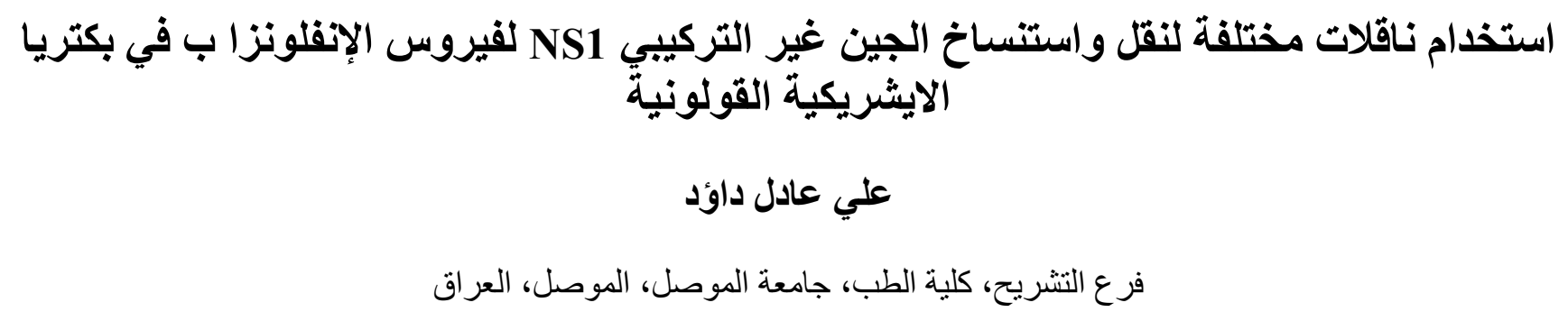

يعتبر مرض الأنفلونزا مرضيًا شائعًا ومُعدٍٍ بسبب فيروسات الأنفلونزا نوع أو أو و ب و و سي. كان الهدف من هذه الدراسة هو البحث في

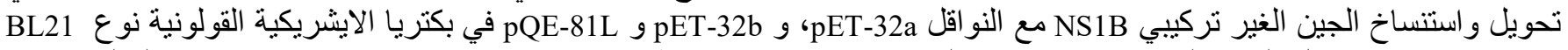

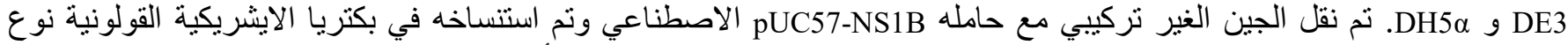

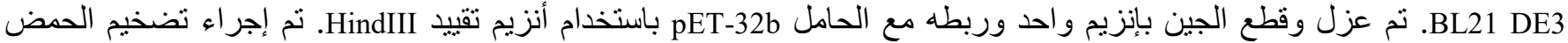
النووي المؤتلف باستخدام تقنية تفاعل البلمرة المتسلسل PCR التقليدي بعد النقل. تم الفحص باستخدام IPTG للمستعمر ات اتلبعل البكتيرية. تمت

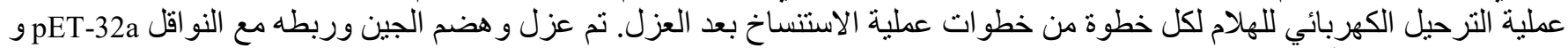

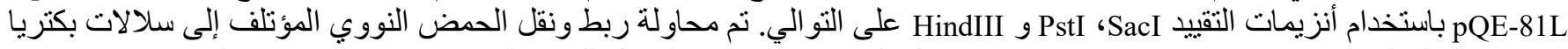

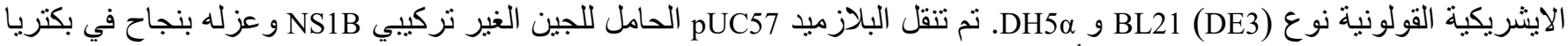

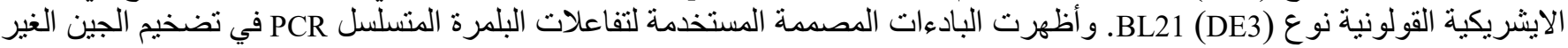




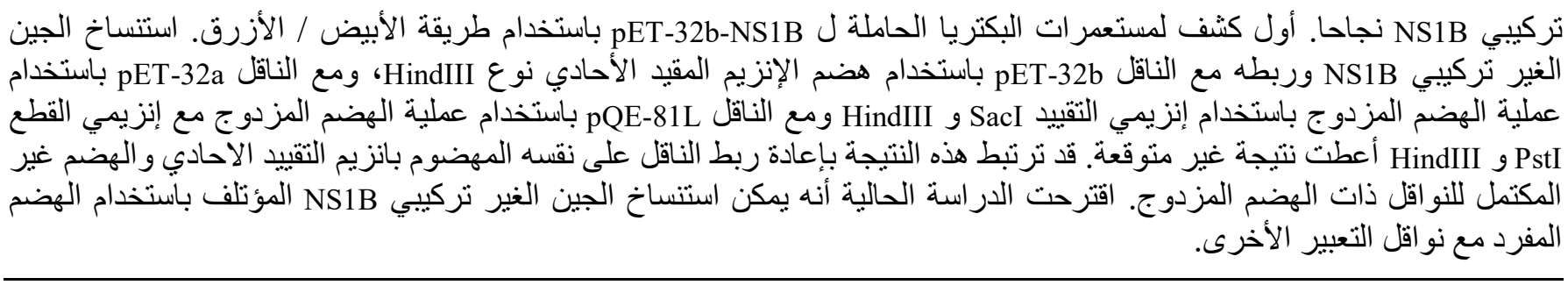

\section{Introduction}

Influenza B virus is an envelope virus, belonging to the family Orthomyxoviridae. Common cold is caused by influenza B and is milder than type A virus. However, some cases lead to severe diseases but rarely morbidity (1). Influenza B viruses lead to seasonal epidemics due to mutations in the viral surface glycoproteins. The influenza nonstructural B protein comprises of 281 amino acids (2). NS1 protein persists only in the infected animal cells and not in vaccinated animals (3). Hence, this may path the way of utilizing NS1 protein as a target for diagnostic of influenza viruses. NS1 protein is involved in virulence and inhibition of the $\alpha / \beta$ interferon system during virus infection. It was shown that, the expression of the NS1B protein complements the growth of influenza A virus with NS1A deleted (4). NS1 gene from various influenza viruses had been cloned. This gene was successfully expressed in Escherichia coli (5). This study describes transformation and cloning of NS1B gene of human influenza B virus in $E$. coli BL21 (DE3) and DH5 $\alpha$ using three types of vectors.

\section{Materials and methods}

All restriction enzymes, DNA polymerase were purchased from Promega. Ligase enzymes were obtained from Yeastern Biotech. Primers used for PCR amplification were synthesized by 1 st Base Laboratories, Kuala Lumpur, Malaysia. Plasmid miniprep and gel extraction kits were purchased from GeneAll and QIAGEN. The pET and pQE vectors were ordered from Novagen and QIAGEN, respectively.

Transformation pUC57-NS1B plasmid into E. coli BL21 (DE3) competent cells was cultured overnight in Luria Bertani (LB) broth containing $100 \mu \mathrm{g} / \mathrm{ml}$ ampicillin at $37^{\circ} \mathrm{C}$ with shaking at $200 \mathrm{rpm}$. The plasmids were isolated using plasmid miniprep kit from GeneAll- South Korea. The products were detected using gel electrophoresis (BIO-RAD) and viewed on a UV transilluminator (GeneFlash) (6). Single restriction digestion was done for pUC57-NS1B and pET-32b by using HindIII enzyme which recognizes 5'A $\boldsymbol{\nabla}$ AGCTT3' sequence. The DNA concentration and purity were measured by NanoDrop Spectrophotometer from (NanoDrop Technologies) using $1 \mu \mathrm{l}$ of the sample. NS1B and digested pET-32b with HindIII were ligated using conventional PCR (Biocompare) (Table 1).

Table 1: Single digestion components in PCR tube using HindIII

\begin{tabular}{lccc}
\hline pUC57-NS1B & $16.8 \mu 1$ & pET-32b & $16.8 \mu 1$ \\
Acetylated BSA & $0.2 \mu 1$ & Acetylated BSA & $0.2 \mu 1$ \\
Buffer E & $2 \mu 1$ & Buffer E & $2 \mu 1$ \\
HindIII enzyme & $1 \mu \mathrm{l}$ & HindIII enzyme & $1 \mu 1$ \\
\hline Total & $20 \mu \mathrm{l}$ & & $20 \mu 1$ \\
\hline
\end{tabular}

The PCR process was initiated with initial denaturation step at $94{ }^{\circ} \mathrm{C}$ for 5 minutes. The process continued with the annealing at $50{ }^{\circ} \mathrm{C}$ for $30 \mathrm{~second}$ and extension step at $72{ }^{\circ} \mathrm{C}$ for 7 minutes. The PCR products were run on gel electrophoresis, then purified by gel extraction (QIAGEN gel extraction kit) to remove the buffer and restriction enzymes. The products were detected using gel electrophoresis. The same protocol was used to isolate pQE-81L from E. coli DH5 $\alpha$. NS1B amplified gene and pQE-81L were digested using PstI and HindIII restriction enzymes (7) (Table 2).

Table 2: Double digestion components in PCR tube using PstI and HindIII

\begin{tabular}{lc}
\hline pQE-81L or NS1B gene & $15.8 \mu 1$ \\
Acetylated BSA & $0.2 \mu 1$ \\
10X Multicore enzyme buffer & $2 \mu 1$ \\
HindIII enzyme & $1 \mu 1$ \\
PstI enzyme & $1 \mu 1$ \\
\hline Total & $20 \mu 1$ \\
\hline
\end{tabular}

PstI enzyme recognizes the 5 'TGCA $\boldsymbol{\nabla}$ G3' sequence and HindIII enzyme recognizes 5'A $\boldsymbol{\nabla}$ AGCTT 3' sequence. After transformation, colony PCR was carried out with pQEfor (5'- CGGATAACAATTTCACACAG-3') and pQErev (5'- TTCTGAGGTCATTACTGG-3') primers. Chelex 100 resin is highly purified, and most suitable for DNA applications was used for detecting bacteria colonies. With the same protocol, pET-32a was digested with SacI and HindIII restriction enzymes for the third attempt (Table $3)$. 
Table 3: Double digestion components in PCR tube using SacI and HindIII

\begin{tabular}{lc}
\hline pET-32a or NS1B gene & $15.8 \mu \mathrm{l}$ \\
Acetylated BSA & $0.2 \mu 1$ \\
10X Multicore enzyme buffer & $2 \mu 1$ \\
HindIII enzyme & $1 \mu 1$ \\
SacI enzyme & $1 \mu 1$ \\
\hline Total & $20 \mu 1$ \\
\hline
\end{tabular}

PCR amplification was done on pUC57-NS1B to amplify NS1B gene with a newly designed primers NS1BforSacI (5'-GAGCTCATGGCGAACAAT-3') and NS1BrevHindIII (5'-AAGCTTCTAATTGTCTCC-3') which introduced SacI and HindIII restriction sites to NS1B for directional cloning of the gene into pET-32a. The HindIII enzyme recognizes 5'A $\boldsymbol{\nabla}$ AGCTT3' sequence while SacI enzyme recognizes 5' GAGCT $\mathbf{\nabla}$ C 3' sequence. The gene was extracted, double digested with SacI and HindIII and used for cloning into pET-32a. pET-32a vector's universal primers, namely $\mathrm{T} 7$ promoter (5'TAATACGACTCACTATAGGG-3') and T7 terminator (5'GCTAGTTATTGCTCAGCGG-3'). The whole investigated clones were 72 clones used in our study for all vectors.

\section{Results}

Transformation of pUC57-NS1B synthetic gene constructs into competent E. coli BL21 (DE3) was successfully done. The presence of pUC57-NS1B was verified by agarose gel electrophoresis. The restriction digestion of pUC57-NS1B was successful and NS1B gene $(870 \mathrm{bp}$ ) was separated and this is shown in Figure 1. pET$32 \mathrm{~b}$ was also linearized and its presence was verified by the band of $5.9 \mathrm{~kb}$ in Figure 1.

Double digestion was done for $\mathrm{pQE}-81 \mathrm{~L}$ by using PstI and HindIII enzymes. As for pUC57-NS1B, it was first digested with HindIII enzyme and the result is shown in Figure 2.

Isolation and digestion of the vector and gene. Lane 1 and 2 shows the successful digestion of pUC57-NS1B with HindIII enzyme and the band of NS1B is of correct size (870bp). Lane 4 shows the double digestion of pQE-81L by using PstI and HindIII and the band is of correct size $(4.8 \mathrm{~kb})$ but in this way still unknown whether the digestion correct complete or not (Figure 2). Plasmid miniprep for both pUC57- NS1B and pQE-81L were verified in lane 3 and 5. However, all the colonies screened gave negative result (Figure 3). The size of amplified band is smaller than $300 \mathrm{bp}$, indicating that only the pQE- $81 \mathrm{~L}$ vector was transformed to cells without the cloning of NS1B gene.
The right band size to be expected is nearly $1000 \mathrm{bp}$. The amplified NS1B gene is shown in figure 4 .

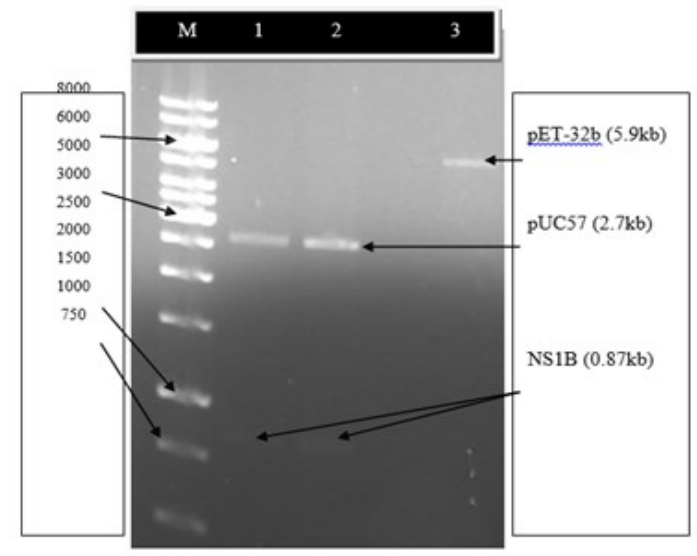

Figure 1: Restriction digestion by HindIII. M: $1 \mathrm{~kb}$ Marker, Lane 1, 2: pUC57 and NS1B gene, Lane 3: pET-32b.

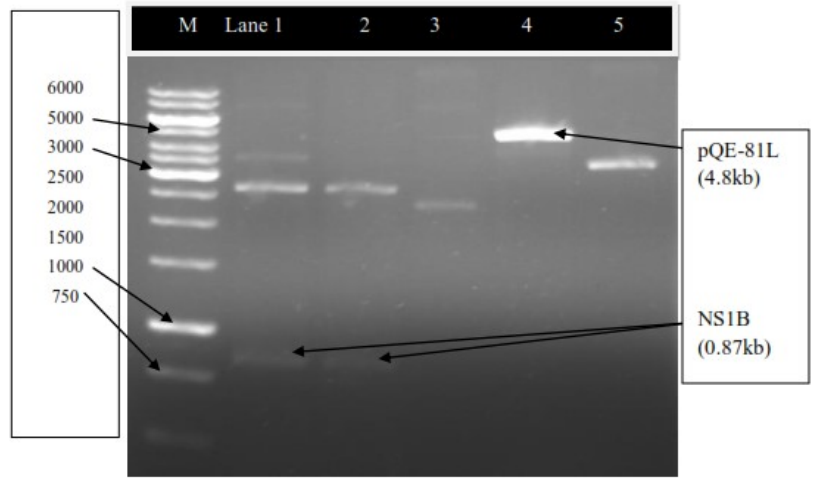

Figure 2: Plasmid isolation and digestion of pUC57-NS1B and pQE-81L M: $1 \mathrm{~kb}$ Marker, Lane 1 and 2: pUC57NS1B digested with HindIII, Lane 3: Miniprep of pUC57-NS1B, Lane 4: Double digestion of pQE-81L, Lane 5: Miniprep of pQE-81L.

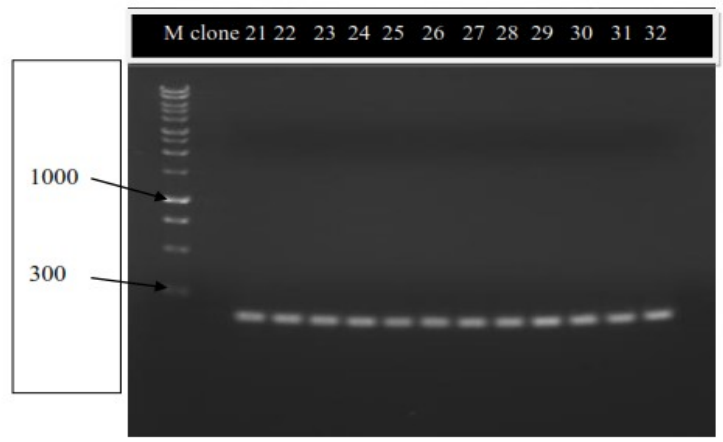

Figure 3: PCR of recombinant pQE-81L-NS1B. 


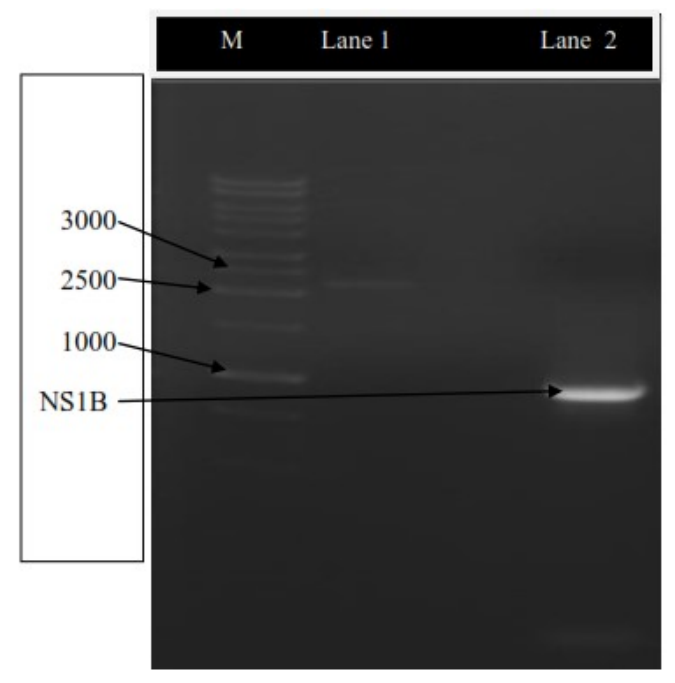

Figure 4: Miniprep of pUC57-NS1B and PCR amplification of NS1B. M: $1 \mathrm{~kb}$ marker, Lane1: Miniprep of pUC57NS1B, Lane 2: PCR amplification of NS1B gene.

The colonies were screened by PCR. All colonies screened gave negative result. As shown in Figure 6, the size of amplified band is smaller than $750 \mathrm{bp}$, indicating that only the pET-32a vector was transformed into the cells without the cloning of NS1B gene. The right band size to be expected is nearly $1500 \mathrm{bp}$ (Figure 5).

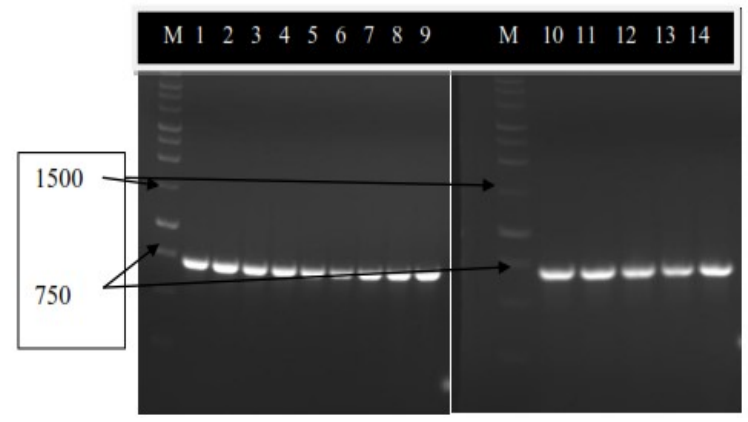

Figure 5: PCR of recombinant pET-32a-NS1B M: $1 \mathrm{~kb}$ Marker, Lane 1-14: Clone from 62-75.

\section{Discussion}

Flu is a viral disease of global importance, it is caused by the influenza virus, which is an RNA virus diversity. NS1 protein persists only in the infected animal cells and not in vaccinated animals, so that, the vaccinated animals are not producing NS1 protein specific antibodies and this allows differentiation between vaccinated and natural infected animals (3). The function of NS1 protein during viral multiplication remains unclear, although a possible regulatory role in viral replication has been suggested from studies of temperature sensitive mutant of NS1 (8). First, the NS1B protein may play an important role in counteracting the activity of antiviral gene products that are expressed in vero cells in an IFN-independent manner (9). NS1 also can suppression of host immune responses such as the apoptotic pathways and activation of phosphoinosidine Kinase-3 (1).

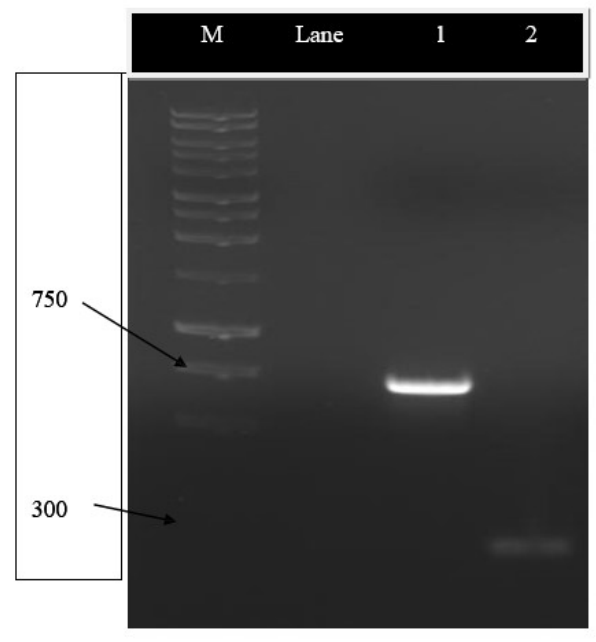

Figure 6: PCR amplification M: $1 \mathrm{~kb}$ Marker, Lane 1: fragment without gene insert from pET-32aNS1B, Lane 2: fragment without gene insert from $\mathrm{pQE}-81 \mathrm{~L}-\mathrm{NS} 1 \mathrm{~B}$.

NS1 gene was isolated from equine influenza virus A/equine 2/Suffolk /89(H3N8), and cloned into pGEX-3X vector then transformed in $E$. coli TG1 competent cell (10). pET-28 vector was employed to express the NS1 gene under the control of the T7 promoter and fused with His-Tag sequences (3). pQE-80L, the other type of vector with 6xHis-tag, was used to express NS1 protein of influenza $\mathrm{A} /$ Chicken/TH/KU14/04 (H5N1) (6). Other study showed that western blot assay for the rNS1 protein, can be applied in a DIVA strategy test and titer of NS1 antibody was low in bird's sera (11). Marzieh found that NS1 gene of influenza A H1N1 virus could be cloned and the rNS1 protein could be expressed using a bacterial protein translation system (12). It has been showed that multifunctional virulence factor heavily after isolation in comparison to FLUAV NS1protein (13). M2e-HA2 sequence of influenza A was cloned into pGS-21a vector to produce GST fusion protein (14).

Currently study, we have successful transformed pUC57-NS1B plasmid into E. coli BL21 (DE3). NS1B gene was successful isolated from the plasmid and cloned with PCR showed in figure 6. Designed primers used for PCR of NS1B showed successful amplification. First screening of pET-32b-NS1B colonies using white/blue method gave 
negative result. Similar negative results were observed when screening colonies of pQE-81L-NS1B, and pET-32aNS1B. Screening of colonies for pET-32a-NS1B showed a band of the size nearly $750 \mathrm{bp}$, which is not as the expected band size of $1500 \mathrm{bp}$. Screening of colonies for all vectors used showed only plasmids without any insertion of the NS1B gene. Cloning NS1B into pET-32b using single restriction digestion with HindIII, pQE-81L using double restriction digestion with (PstI and HindIII), pET-32a using double restriction digestion with (SacI and HindIII), gave unexpected result. This result may relate to re-ligation of digested vector for single digestion and uncompleted digestion for vectors of double restriction digestion.

A further construction of NS1 gene into pET32a, pET-32b, and pQE-81L vectors should be performed with best precaution of procedures to avoid any contamination and the use of appropriate restriction enzymes with high efficiency to get recombinant construction. The NS1B gene can also be cloned into other expression vectors according to the open reading frame and use other host.

Previous studies was isolated NS1 gene from equine influenza virus A/equine 2/Suffolk /89(H3N8), cloned into pGEX-3X vector and transformed in E. coli TG1 competent cell (10). NS1 gene from Influenza A (H9N2) was cloned and highly expressed using pET-28 vector (3). pQE-80L, the other type of vector with 6xHis-tag, was used to express NS1 protein of influenza A/Chicken/TH/KU14/04 (H5N1) (6). Very limited studies have isolated NS1B gene using vectors other than those used in this study.

This study was faced some limitations. First, several repetitions of digestion and ligation of enzyme are more fastidious. Second, difficult insertion of plasmids in competent host cells. Furthermore, choosing one appropriate vector is better than several vectors with single enzyme digestion. It is suggested that the overexpression of the constructed recombinant into expression system are being studied and work on purification can also be performed using immobilized affinity chromatography resin.

\section{Conclusion}

According to the results of this study, it could be concluded that the NS1B gene was successful transformed, cloned and isolated from E. coli BL21 (DE3). Transformation, cloning and isolation of pET32a, pET-32b, pQE-81L were successful done in E. coli. This study was failed for ligation of NS1B gene in the expressing vectors.

\section{References}

1. Marzieh S, Mojgan B, Fatemeh Y, Vahidreza Y, Elham T, Bahram K. Cloning and expression of influenza H1N1 NS1 protein in Escherichia coli BL21. Iran J Biotechnol. 2014; 12(1):12625: 1-5 DOI: $10.5812 / \mathrm{JJB} .12625$.

2. Manasatienkij W, Lekcharoensuk P, Upragarin N. Expression and purification of NS1 protein of highly pathogenic avian influenza virus H5N1 in Escherichia coli. Kasetsart J Nat Sci. 2008; 42:485-494.

3. Fang KW, Xio FY, Yi CW, Cun Z, Li HX, Si DL. Cloning, prokaryotic expression, and antigenicity analysis of $111 \mathrm{NS} 1$ gene of H9N2 swine influenza virus. Sci Rep. 2016; 6:895-900.

4. Donelan N, Dauber B, Wang X, Basler C, Wolff T, Garcia SA. The $\mathrm{N}$-and C- terminal domains of the NS1 protein of influenza B virus can independently inhibit IRF-3 and beta interferon promoter activation. J Virol. 2004; 78(21):11574-82, DOI: 10.1128/JVI.78.21.11574-11582.

5. Hatada E, Takezawa T, Fukuda R. Specific binding of influenza A virus NS1 protein to the virus minus-sense RNA in vitro. J Gen Virol. 1992; 73(1):17-25, DOI: 10.1099/0022-1317-73-1-17

6. Manasatienkij W, Lekcharoensuk P, Upragarin N. Expression and purification of NS1 protein of highly pathogenic avian influenza virus H5N1 in Escherichia coli. Kasetsart J Nat Sci. 2008; 42:485-494.

7. Masoud M, Mehdi G, Hadi T, Reza G. Cloning, expression and purification of M2e-HA2 from Influenza A virus in Escherichia coli. J Vet Res. 2015; 19(2):124-129.

8. Binns M, Ely. Expression of the non-structural protein NS1 of influenza virus and detection of anti-NS1 antibody in serum. European Patent Application. 1996; 33, no: 96300681.2:1-20.

9. Dauber B, Heins G, Wolff T. The Influenza B virus nonstructural NS1 protein is essential for efficient viral growth and antagonizes beta interferon induction. J Virol. 2003; 78(4): 1865-1872, DOI: 10.1128/JVI.78.4.1865-1872.

10. Birch Ma I, Rowan A, Jane PJ, Mumford J, Binns M. Expression of the nonstructural protein NS1 of equine influenza a virus: detection of Anti-NS1 antibody in post infection equine sera. J Virol Methods. 1997; 65(2):255-263, DOI: 0166-0934.

11. Ramin S, Mehdi VM, Mohammad BHS, Farhid H. The Cloning of non-structural-1 (NS1) gene of H9N2 subtype of avian influenza virus in pGEX-4T-1 and pMAL-c2X plasmids and expression in Escherichia coli DH5 $\alpha$ strain. Adv Biosci Biotechnol. 2012; 3:283289, DOI:10.4236/abb.2012.33040.

12. Muhammad J, Muhammad HZ. Molecular cloning and expression of NS1 of the influenza "A" virus from different subtypes, master project, School of Life Sciences, Sodertorns University; 2011.

13. Patzina $\mathrm{C}$, Botting $\mathrm{CH}$, García SA, Randall RE, Hale BG. Human interactome of the influenza B virus NS1 protein. J Gen Virol. 2017; 98:2267-2273, DOI:10.1099/jgv.0.000909.

14. Li ZF, Liu Y, Rui Y, Ke FS, Jin LY. Cloning and phylogenetic analysis of NSI Gene of H3N2 subtype swine influenza virus from different isolates in chongqing china 2010-2011. J Anim Vet Adv. 2012; 11(12):2126-2128, DOI: 10.3923/javaa.2012.2126.2128. 